\title{
Application of Functionalized Lanthanide-based Nanoparticles for the Detection of Okadaic Acid-specific Immunoglobulin G
}

Filip Stipić, ${ }^{a}$ Galja Pletikapić, ${ }^{b}$ Željko Jakšić, ${ }^{a}$ Leo Frkanec$^{c},{ }^{c}$ Goran Zgrablić, ${ }^{d}$ Petra Burić, ${ }^{a}$ Daniel M. Lyons**a

${ }^{a}$ Center for Marine Research, Ruđer Bošković Institute, G. Paliaga 5, 52210 Rovinj, Croatia.

${ }^{\mathrm{b}}$ Department of Marine and Environmental Research, Ruđer Bošković Institute, Bijenička cesta 54, 10000 Zagreb, Croatia.

${ }^{\mathrm{c}}$ Department of Organic Chemistry and Biochemistry, Ruđer Bošković Institute, Bijenička cesta 54, 10000 Zagreb, Croatia.

d Time Resolved X-Ray Spectroscopy Laboratory, Elettra-Sincrotrone Trieste, 34149 Basovizza, Italy.

* to whom correspondence may be addressed: E-mail: lyons@irb.hr, Fax: +385 52 804780; Tel: +38552804725 


\begin{abstract}
Marine biotoxins are widespread in the environment and impact on human health via contaminated shellfish, causing diarrhetic, amnesic, paralytic or neurotoxic poisoning. In spite of this methods for determining if poisoning has occurred are limited. We show the development of a simple and sensitive luminescence resonance energy transfer (LRET) -based concept which allows the detection of anti-okadaic acid rabbit polyclonal IgG (mouse monoclonal IgG1) using functionalized lanthanide-based nanoparticles. Upon UV excitation, the functionalized nanoparticles were shown to undergo LRET with fluorophore-labeled anti-okadaic acid antibodies which had been captured and bound by okadaic acid-decorated nanoparticles. The linear dependence of fluorescence emission intensity with antigen-antibody binding events was recorded in the nanomolar to micromolar range, while essentially no LRET signal was detected in the absence of antibody. These results may find applications in new, cheap and robust sensors for detecting not only immune responses to biotoxins but to a wide range of biomolecules based on antigen-antibody recognition systems. Further, as the system is based on solution chemistry it may be sufficiently simple and versatile to be applied at point-of-care.
\end{abstract}

\title{
Keywords
}

biotoxin, immunodetection, diagnostics, biosensor, point-of-care 


\section{Introduction}

Biosensors, based on the detection of targets using biomolecules, have become commonplace in today's world. Such sensors, employing various physiologically-relevant biomolecules have found widespread use in diverse areas, ranging from pathogen detection to diagnosis and tracking of disease progression in humans. ${ }^{1}$ While many biosensors have traditionally focused on the use of antibodies for antigen detection, the detection of antibodies is increasingly recognized as an important aspect of biosensor research. Antibodies may be used as biomarkers for following a range of illnesses including tuberculosis, ${ }^{2}$ human papillomavirus, ${ }^{3}$ and breast cancer. ${ }^{4}$ In addition, various conditions such as inflammatory bowel disease or HIV infection ${ }^{5}$ may also be followed based on tracking changes to systemic antibody response to gut microbes when the latter's balance is disrupted as a consequence of the disease. Further, determination of antibody presence and concentration provide valuable information on the efficacy of vaccines in inducing an immune response against specific targets such as, for example, malaria. ${ }^{6}$

In recent years a range of techniques has been developed for the detection and quantification of antibodies against specific antigens, peptides, proteins and various biomolecules. Primary among these are methods derived from enzyme-linked immunosorbent assay (ELISA) in which a complementary biomolecule to the antibody is immobilized on a substrate and, upon binding of the antibody, may be labeled with a fluorescent dye which is interrogated to determine its concentration and by extension the concentration of antibody present. This method has been applied to the detection of a broad spectrum of antibodies ranging from those against Helicobacter pylori ${ }^{7}$ to markers in rheumatoid arthritis, ${ }^{8,9}$ lupus ${ }^{10}$ and heparin-induced thrombosis. ${ }^{11}$ Other related fluorescent marker approaches include, for example, immunoprecipitation assays and recombinant immunofluorescence assays for detection of 
antibodies to aquaporin-4 in patients with neuromyelitis optica. ${ }^{12,13}$ Increasingly, these sensing techniques are being developed as antigen-bound micro-arrays in which large numbers of antigens are anchored to a substrate to show good sensitivity and low detection limit due to a high fluorescence signal, or a combination of antigens are bound providing a multiparametric sensor for a range of antibodies. ${ }^{10,14}$

An alternative highly sensitive approach to antibody detection and quantification may potentially be based on exploiting resonance energy transfer, a technique based on the excitedstate non-radiative energy transfer between specific donor-acceptor pairs due to dipole-dipole interactions and spatial proximity, with the sensitivity of the technique down to the single target molecule level already having been shown. ${ }^{15}$ In fact, the use of fluorescence resonance energy transfer from functionalized terbium chelates to fluorescent organic dyes based on antibodyantigen binding for the detection of total prostate specific antigen has recently been reported. ${ }^{16} \mathrm{~A}$ different concept based on the forced separation of a donor/acceptor pair upon binding to an anti-HIV-1-p17 antibody leading to FRET quenching hence allowing antibody concentration estimation was also recently reported. ${ }^{17}$

Further development of these fluorescence techniques may be achieved through taking advantage of the unique optical properties of nano-sized materials. Application of lanthanidebased nanoparticles as donors in such types of assay (luminescence resonance energy transfer; LRET) may be promising for a number of reasons including large Stokes' shifts, thus avoiding direct excitation of the acceptor, and reduction of background emission in some applications. ${ }^{18}$ Further, they have high quantum yields and very narrow emission bands compared to organic fluorophores, and long-lived excited state lifetimes ( $\sim 1 \mathrm{~ms})$ enable both facile measurement of donor lifetime changes and elimination of short-lived background auto-fluorescence by signal 
collection after specific time delays. ${ }^{19,20}$ Indeed, detection of resonance energy transfer at the single lanthanide nanoparticle level has also been reported. ${ }^{21}$ Thus, biosensors based on nanomaterials may offer significant advantages over those currently in use in terms of sensitivity. The application of such a fluorescence technique, based on straightforward mix-and-measure solution chemistry, holds the promise for simple, rapid and cheap biosensors that may be applied at point-of-care. In this direction we show herein the ability to detect specific antibodies arising from exposure to the environmental biotoxin okadaic acid, a potent serine/threonine protein phosphatase $2 \mathrm{~A}$ inhibitor and tumor promoter, ${ }^{22-24}$ by concentration-dependent resonance energy transfer in what may be a useful new method in the analytical chemist's toolbox for the development of point-of-care diagnostic sensors.

\section{Experimental Methods}

Lanthanum nitrate and terbium nitrate (99.99\%) were obtained from Sigma while cerium nitrate $(99.9 \%)$ was supplied by Fluka. Sodium fluoride (p.a.) was received from Kemika, and ammonium hydroxide (ACS, 28.8-30\% $\mathrm{NH}_{3}$ basis) and O-phosphorylethanolamine from Sigma. For all aqueous solutions, ultrapure water $(18 \mathrm{M} \Omega)$ was used. Rabbit polyclonal antibodies to okadaic acid were obtained from Abcam (Cambridge, UK) while goat anti-rabbit IgG $\mathrm{F}_{\mathrm{ab}}$ and AlexaFluor 488 fluorescent dye were supplied by Life Technologies.

\section{Synthesis of $\mathrm{LaF}_{3}: \mathrm{Ce}, \mathrm{Tb}$ nanoparticles}

All precursor materials were obtained at the highest possible purity and used as received (supplementary information, SI). The synthesis of water soluble and amino-capped $\mathrm{La}_{0.40} \mathrm{~F}_{3}: \mathrm{Ce}_{0.45}, \mathrm{~Tb}_{0.15}$ nanoparticles (LaNPs) was carried out based on the method of Diamente et al. ${ }^{25}$ with some small modifications. Briefly, to a $\mathrm{NH}_{4} \mathrm{OH}-$ neutralized aqueous solution $(25 \mathrm{~mL})$ of $144 \mathrm{mg}$ ( $1 \mathrm{mmol})$ O-phosphorylethanolamine was added $130 \mathrm{mg}$ (3 mmol) NaF. The solution 
was heated to $40^{\circ} \mathrm{C}$ upon which a solution of $\mathrm{La}\left(\mathrm{NO}_{3}\right)_{3} \cdot 6 \mathrm{H}_{2} \mathrm{O}(540 \mathrm{mg}, 1.26 \mathrm{mmol})$, $\mathrm{Ce}\left(\mathrm{NO}_{3}\right)_{3} \cdot 6 \mathrm{H}_{2} \mathrm{O}(580 \mathrm{mg}, 1.34 \mathrm{mmol})$ and $\mathrm{Tb}\left(\mathrm{NO}_{3}\right)_{3} \cdot 6 \mathrm{H}_{2} \mathrm{O}(240 \mathrm{mg}, 0.56 \mathrm{mmol})$ in $2 \mathrm{~mL}$ ultrapure water was added drop wise. The solution was held at $40^{\circ} \mathrm{C}$ overnight upon which the nanoparticles were precipitated with acetone, isolated by centrifugation (10 min at $3000 \mathrm{rpm})$, washed with acetone and centrifuged again.

Covalent conjugation of okadaic acid to $\mathrm{LaF}_{3}: \mathrm{Ce}, \mathrm{Tb}$ nanoparticles

Okadaic acid was first activated by reaction with N-hydroxysuccinimide (NHS) and N-(3dimethylaminopropyl)-N`-ethylcarbodiimide hydrochloride (EDAC). NHS (12 mg) and EDAC (19 mg) were dissolved in $1 \mathrm{~mL}$ 2-(N-morpholino)ethanesulfonic acid (MES) buffer (100 mM, $\mathrm{pH}$ 6.0). A $20 \mu \mathrm{L}$ aliquot was withdrawn and further diluted five-fold in MES buffer, followed by the addition of $200 \mu \mathrm{L}$ of the okadaic acid solution $\left(1 \mu \mathrm{g} \mathrm{mL} \mathrm{m}^{-1}, 80 \%\right.$ methanol). The mixture was stirred for $1 \mathrm{~h}$ at $25^{\circ} \mathrm{C} .100 \mu \mathrm{g}$ lanthanide nanoparticles were dispersed in $700 \mu \mathrm{lof} 50 \mathrm{mM}$ borate buffer solution, $\mathrm{pH} 8.0$ and the $300 \mu \mathrm{L}$ of activated okadaic solution was added. The final concentration of nanoparticles was $100 \mu \mathrm{g} \mathrm{mL}^{-1}$ and okadaic acid was $200 \mathrm{ng} \mathrm{mL}^{-1}$. The mixture was stirred for $2 \mathrm{~h}$ at $25^{\circ} \mathrm{C}$. (Note that contact with okadaic acid may cause severe diarrhea, nausea and vomiting, thus requiring appropriate protection measures to be taken.)

\section{Antibody labeling and binding to okadaic acid-decorated $L a F_{3}: C e, T b$ nanoparticles}

In a typical experiment, anti-okadaic acid rabbit polyclonal IgG was labeled with goat antirabbit IgG $\mathrm{F}_{\mathrm{ab}}$ fragment conjugated with AlexaFluor 488 using the Zenon Rabbit IgG labeling kit following the manufacturer's protocol. Briefly, an aliquot of $1 \mu \mathrm{L}$ rabbit polyclonal IgG was diluted with $9 \mu \mathrm{L}$ phosphate-buffered saline (PBS), $\mathrm{pH} 7.6$, and mixed with $5 \mu \mathrm{L}(1 \mu \mathrm{g})$ goat anti-rabbit IgG $\mathrm{F}_{\mathrm{ab}}$ fragment conjugated with AlexaFluor488 (AF488) fluorescent dye (in PBS buffer solution) following the manufacturer's protocol. After labeling, this solution was added to 
the dispersion of okadaic acid-decorated LaNPs and gently mixed for $30 \mathrm{~min}$ at $25^{\circ} \mathrm{C}$. The fluorescence spectrum was measured (Scheme 1), upon which further aliquots of rabbit serum containing IgG anti-okadaic acid antibodies were sequentially added to the solution, incubated and the fluorescence measurement repeated.

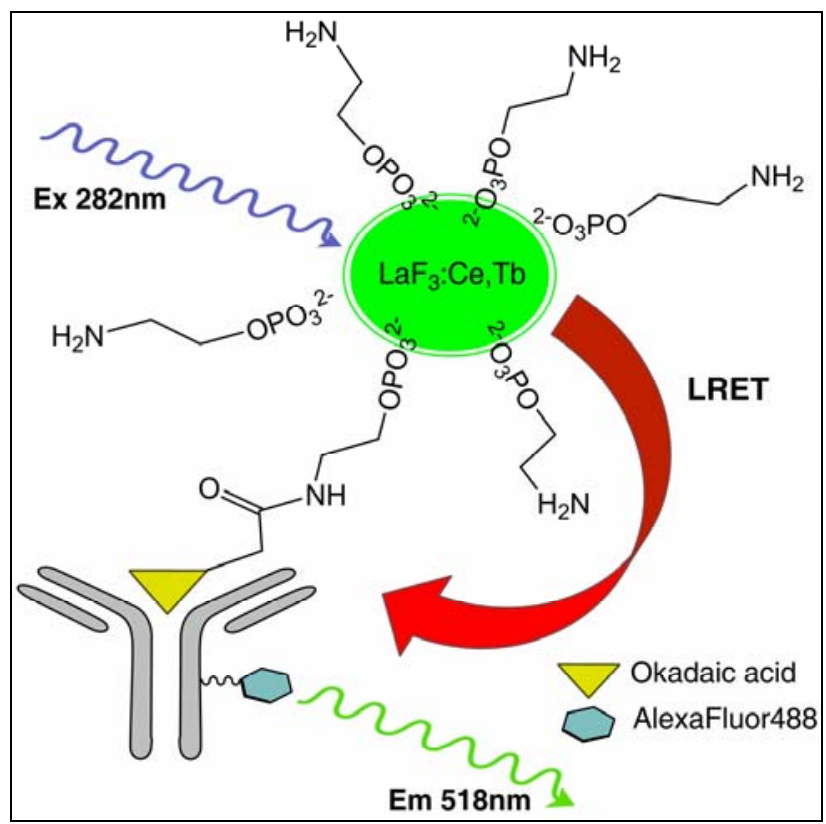

Scheme 1 Luminescence resonance energy transfer between LaNP donor and AlexaFluor488 acceptor upon okadaic acid-antibody binding

\section{Instrumentation}

X-ray diffraction data were collected on a Rigaku Ultima IV multi-purpose diffractometer in parafocusing geometry, operating at $40 \mathrm{kV}$ and $40 \mathrm{~mA}$, and using $\mathrm{Cu} \mathrm{K} \alpha$ radiation of wavelength $1.5418 \AA$, scan speed of $1^{\circ} \min ^{-1}(2 \theta)$ and step size of $0.02^{\circ}(2 \theta)$. Data were processed on Rigaku PDXL proprietary software. Atomic force microscopy was performed on samples deposited from borate buffer solution $(\mathrm{pH} 8,0.05 \mathrm{M})$ on freshly cleaved mica surfaces using a Multimode AFM, with Nanoscope IIIa controller (Bruker, Billerica USA) with a vertical engagement (JV) $125 \mu \mathrm{m}$ scanner, in tapping mode using silicon tips (RTESP, Bruker, tip radius 
nom. $8 \mathrm{~nm}$ ). Processing and analysis of images was done on NanoScope software (Digital Instruments, version V614r1). Luminescence measurements were carried out on a Kontron SFM25 spectrofluorometer and Cary Eclipse fluorescence spectrophotometer at $25^{\circ} \mathrm{C}$ using quartz cuvettes of $1 \mathrm{~cm}$ optical path length, with samples dispersed in borate buffer solution ( $\mathrm{pH}$ 8, $0.05 \mathrm{M})$. Time resolved fluorescence lifetime measurements were recorded using the fourth harmonic of a $1 \mathrm{kHz}$ laser system (Coherent Inc.), consisting of a Ti:SA oscillator (Mira 900) and a Ti:SA amplifier (Legend), at a laser repetition rate of $100 \mathrm{~Hz}$.

\section{Results and Discussion}

The as-prepared dry, powdered nanoparticles gave X-ray diffraction peaks which showed a good match to a hexagonal crystal phase (ICDD card 01-070-3182) of $\mathrm{LaF}_{3}$ (Figure 1). No phase separation of the cerium and terbium dopants was found, while whole pattern fitting with pseudo-Voigt shaped peaks indicated an average crystallite size of $2.6 \mathrm{~nm}$ based on applying the Scherrer formula to the seven main reflections. AFM measurements on LaNPs $(5 \mu \mathrm{L}$ of a borate buffer solution with nanoparticles at a concentration of $20 \mu \mathrm{g} \mathrm{mL}^{-1}$ ), gave an average particle diameter of $23.9 \pm 5 \mathrm{~nm}$ (Figure 2), while dim spots of $<2 \mathrm{~nm}$ size noted on the AFM image are likely excess O-phosphorylethanolamine organic ligand used in the synthesis to maintain small particle sizes and which was not efficiently removed by the washing steps. While solutions of antibodies, LaNPs and okadaic acid-functionalized LaNPs gave stable dispersions individually, the corresponding functionalized LaNP-okadaic acid-antibody complexes showed some agglomeration behavior, with small agglomerates typically ranging in size from about $100-$ $200 \mathrm{~nm}$ by AFM (Figure 3). While unambiguous identification of the components of these small agglomerates is not possible by AFM, their sizes are consistent with dynamic light scattering data where hydrodynamic diameters of $19.4 \mathrm{~nm}$ and $184.9 \mathrm{~nm}$ were noted for nanoparticles and agglomerates, respectively (data not shown). Considering hydrodynamic radii for the $\operatorname{IgG}$ 
antibody, $\mathrm{F}_{\mathrm{ab}}$ fragment and nanoparticle of $5.41 \mathrm{~nm}, 2.91 \mathrm{~nm}$ and $9.70 \mathrm{~nm}$ respectively and taking the average number of antibodies associated with each nanoparticle as 50, the LaNPokadaic acid-antibody complexes would show a hydrodynamic diameter of $71.2 \mathrm{~nm} .^{26,27}$ This gives an indication that agglomerates could consist of 2-3 LaNP-okadaic acid-antibody complexes associated together. The reason for such small agglomerates forming can not be established here but may be related to surface energy considerations and/or mediation by other molecules in solution derived from the rabbit whole serum.

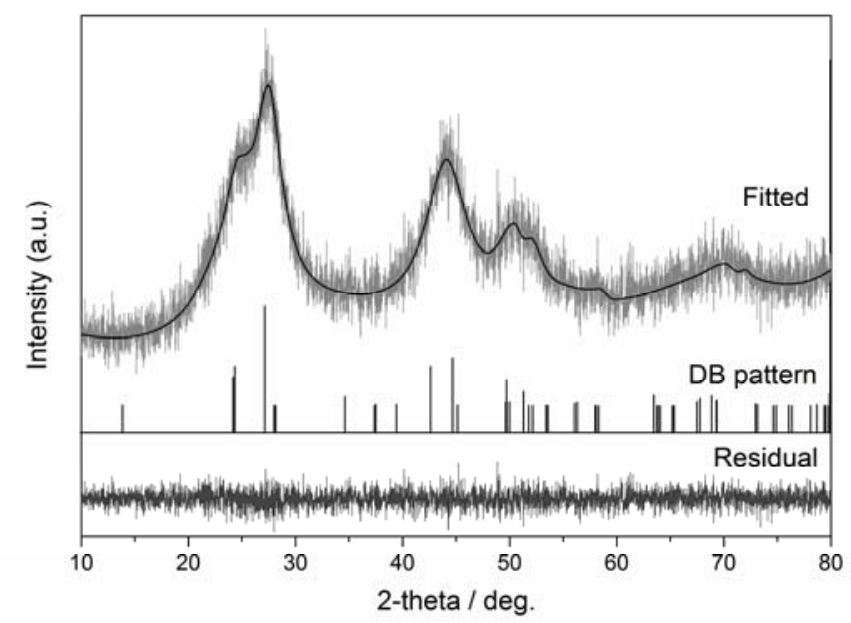

Figure 1 Powder X-ray diffraction data, simulated pattern (Fitted) based on crystallographic database values (DB pattern) and residual plot for $\mathrm{LaF}_{3}: \mathrm{Ce}, \mathrm{Tb}$ nanoparticles

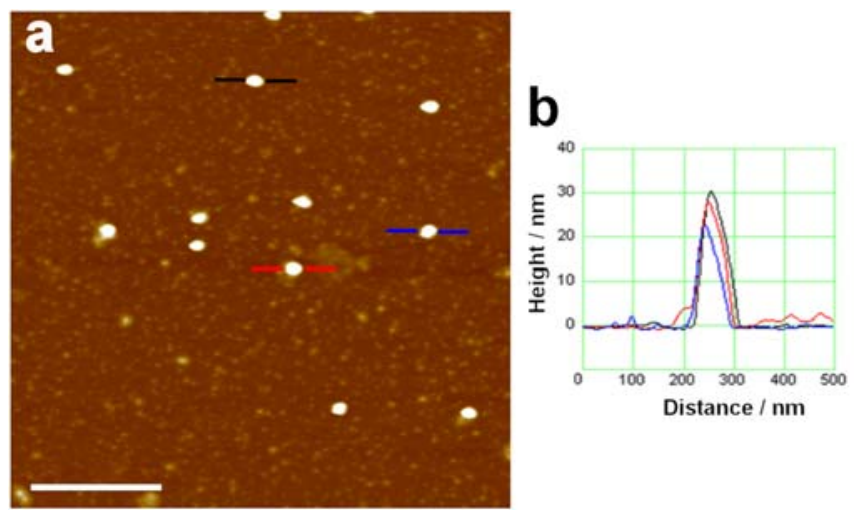

Figure 2 (a) AFM height image of $\mathrm{LaF}_{3}: \mathrm{Ce}, \mathrm{Tb}$ nanoparticles with (b) height analysis along indicated lines (scale bar $=500 \mathrm{~nm}$ ). 

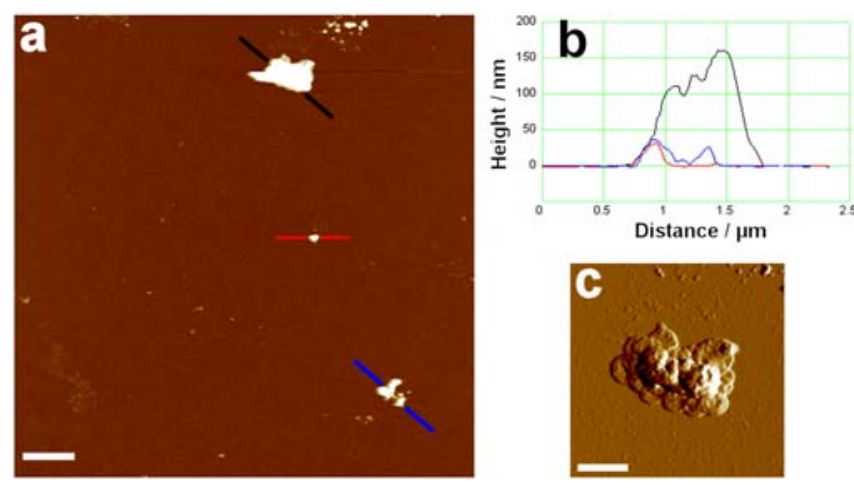

Figure 3 (a) AFM height image of okadaic acid-decorated $\mathrm{LaF}_{3}: \mathrm{Ce}, \mathrm{Tb}$ nanoparticles incubated with $\mathrm{F}_{\mathrm{ab}}$-AlexaFluor488 labeled anti-okadaic acid antibodies (scale bar = $1 \mu \mathrm{m})$ with (b) height analysis along indicated lines. (c) Deflection image of an agglomerate (scale bar $=500 \mathrm{~nm})$.

Excitation and emission spectra for LaNPs and AF488 are given in Figure 4. The LaNP donor and AF488 acceptor excitation bands are widely separated thus minimizing fluorescence due to off-peak excitation of the acceptor. Further, as AF488 has a fast decay lifetime of $4 \mathrm{~ns}$, time gating removes any fluorescence signal arising from direct excitation. The nanoparticle ${ }^{5} \mathrm{D}_{4}$ $\rightarrow{ }^{7} \mathrm{~F}_{6}\left(\mathrm{~Tb}^{3+}\right)$ emission line at $490 \mathrm{~nm}$ shows excellent overlap with the AF488 excitation band centered at $495 \mathrm{~nm}$, while the AF488 emission at $518 \mathrm{~nm}$ does not overlap with other emission lines from the nanoparticle thus indicating the appropriateness of the selected donor-acceptor pair for interrogating antigen-antibody binding by luminescence resonance energy transfer.

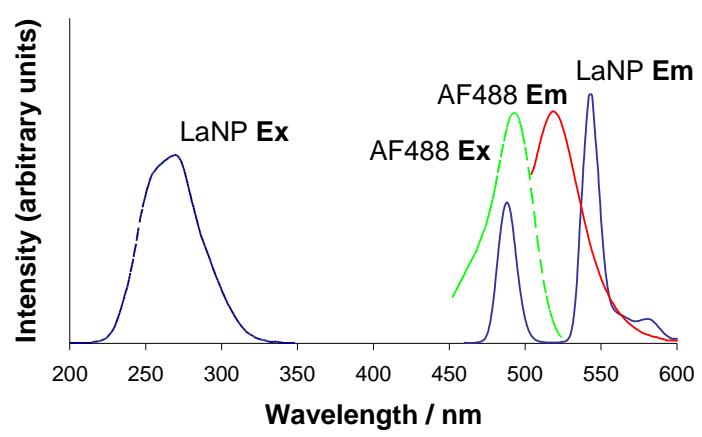

Figure 4 Excitation (Ex) and emission (Em) spectra of $\mathrm{LaF}_{3}: \mathrm{Ce}, \mathrm{Tb}$ nanoparticle (LaNP) donor and AlexaFluor 488 (AF488) acceptor 
The emission spectrum of the LaNP-OA-antiOA-AF488 complex under UV excitation at $282 \mathrm{~nm}$ is shown in Figure 5. With increasing concentrations of labeled antibodies a gradual increase in the intensity of the $518 \mathrm{~nm}$ AF488 emission was noted with a concomitant decrease in intensity of the $490 \mathrm{~nm}$ LaNP emission. The dynamic response in terms of $\mathrm{I}_{518} / \mathrm{I}_{490}$ intensity ratio at peak maximum of these bands showed a linear dependence on antibody concentration, and hence binding events, in the nano- $(83 \mathrm{nM})$ to micromolar $(1.33 \mu \mathrm{M})$ range (Figure 5 , inset) with a limit of detection calculated at $42 \mathrm{nM}$. Indeed, using this ratio reduces or eliminates instrumental counting variability between samples and non-specific sample absorbance based on, for example, turbidity. The donor component is necessarily kept in excess as significant selfquenching would occur for the case where acceptor is in excess. The linear relationship between antibody-antigen binding and fluorescence signal measured here indicates that self-quenching in this LRET system is minimized despite a relatively small Stokes' shift and some overlap between the AF488 excitation and emission bands. Further, any agglomeration- deagglomeration processes for the detection complexes in solution were not found to affect the LRET signal over time.

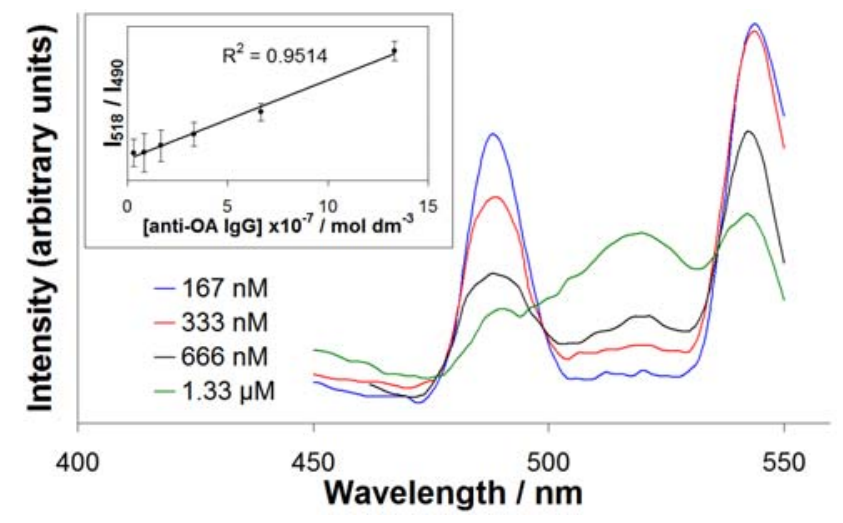

Figure 5 Emission spectra of the LaNP-OA-antiOA antibody-AF488 complex $\left(\lambda_{\text {ex }} 282 \mathrm{~nm}\right)$ for various concentrations of labeled antibodies. Inset: corresponding $\mathrm{I}_{518} / \mathrm{I}_{490}$ intensity ratios at peak maxima for different antibody concentrations 
A corresponding series of control measurements was made for the complex in which no OA had been conjugated to the LaNPs to determine the role of non-specific binding in the LRET process. In the absence of specific antibody-antigen binding no reduction in $490 \mathrm{~nm}$ emission intensity was noted for the LaNPs and no increase in AF488 emission at $518 \mathrm{~nm}$ was found, indicating that achieving successful resonance energy transfer in this system is essentially a function of specific molecular recognition between OA and anti-OA antibodies.

Such an absence, or very low-level, of non-specific binding is surprising although may be related to steric reasons as LRET shows extreme sensitivity to distance. This means that a sufficiently close approach by the AF488 acceptor to the donor may be disfavored due to hindrance by the specific, and perhaps non-random, orientation of anti-OA antibody- $\mathrm{F}_{\mathrm{ab}}$ complex with respect to LaNP as has been noted for other systems. ${ }^{28}$

Further confirmation of resonance energy transfer within the system was obtained through photoluminescence lifetime measurements which, using a longer gated delay of $500 \mu \mathrm{s}$, avoids interference from direct excitation of the AF488 acceptor. For excitation at $266 \mathrm{~nm}$, data showed a mono-exponential decay more than $13 \%$ faster for the LaNP-OA-anti-OA antibody-AF488 complex $(1.91 \mathrm{~ms})$ compared to the LaNP only $(2.20 \mathrm{~ms})$ indicating increased de-excitation efficiency based on energy transfer to the short fluorescence lifetime acceptor.

While much published work has used, for example, streptavidin/avidin-biotin binding for demonstrating proof of concept in resonance energy transfer systems due to their extremely high mutual affinity, ${ }^{29,30}$ whole serum-derived polyclonal antibodies were interrogated in this work as they may be more relevant in a clinical setting. In addition, functionalizing nanoparticles with other OA-group congeners (which have only minor structural changes from the okadaic acid 
used here) would still be expected to give a FRET signal as polyclonal antibodies are able to recognize multiple epitopes within a specific toxin group or class giving better cross-reactivity and ultimately more robust binding ability to the biotoxin. Indeed, the relative ease with which resonance energy transfer was detected in our system, particularly due to the acceptor emission band at $518 \mathrm{~nm}$ not overlapping with other LaNP donor emission lines and hence avoiding the need for peak deconvolution after data acquisition, demonstrates the potential of the technique to detect an immune system response to marine biotoxins. Further, the relative lack of non-specific binding and the linear response in intensity of emission with number of binding events shows promise for the development of such a sensor for these small target biomolecules. This concept allowed a 'test tube' diagnostic design, in this particular case for okadaic acid antibody detection, where all the reactants such as functionalized lanthanide nanoparticles and antibody labels, are present in different solutions and require only simple mixing for binding to occur without additional precipitation of complexes and washing steps such as, for example, in competitive ELISA.

\section{Conclusions}

To our knowledge, this work represents the first use of lanthanide nanoparticle-based luminescence resonance energy transfer to labeled antibodies in order to achieve a robust, simple, sensitive and quantitative 'test tube' method for the detection of antibodies against marine biotoxins. This work represents a step towards the development of an integrated 'mixand-shake' solution-based technique which may be applied at point-of-care and does not need highly trained personnel or expensive specialized laboratory equipment but rather a simple handheld fluorometer. Future work should extend this research to an integrated system in which a LRET signal may be detected, for example, by conformational changes upon antibody-antigen binding which may eliminate the antibody labeling step. We expect our work will stimulate 
further research in the area of physical chemistry to answer important questions, for example determining antigen orientation with respect to the nanoparticle surface and how this modulates its interaction with antibodies, near-surface steric effects and how it affects antibody-antigen binding, impact of tailoring the length and flexibility of the nanoparticle-antigen linkers etc. Ultimately, such research is expected to have a strong impact on developing strategies for tailored biosensors which will find use in the priority areas of point-of-care medical diagnostics, consumer health, and food quality and safety monitoring.

\section{Acknowledgement}

These materials are based on work financed by the Croatian Science Foundation through project $02.05 / 17$. Financial support from the Royal Society of Chemistry and the Adris Foundation is also gratefully acknowledged. V. Svetličić is thanked for assistance with AFM and M. Dutour Sikirić for help with DLS measurements.

\section{References}

1. Perumal, V.; Hashim, U. Advances in Biosensors: Principle, Architecture and Applications. J. Appl. Biomed. 2014, 12, 1-15.

2. Ravindran, R.; Krishnan, V. V.; Khanum, A.; Luciw, P. A.; Khana, I. H. Exploratory Study on Plasma Immunomodulator and Antibody Profiles in Tuberculosis Patients. Clin. Vaccine Immunol. 2013, 20, 1283-1290.

3. Koslabova, E.; Hamsikova, E.; Salakova, M.; Klozar, J.; Foltynova, E.; Salkova, E.; Rotnaglova, E.; Ludvikova, V.; Tachezy, R. Markers of HPV Infection and Survival in Patients with Head and Neck Tumors. Int. J. Cancer 2013, 133, 1832-1839.

4. Anderson, K. S.; Ramachandran, N.; Wong, J.; Raphael, J. V.; Hainsworth, E.; Demirkan, G.; Cramer, D.; Aronzon, D.; Hodi, F. S.; Harris, L. et al. Application of Protein 
Microarrays for Multiplexed Detection of Antibodies to Tumor Antigens in Breast Cancer. J. Proteome Res. 2008, 7, 1490-1499.

5. Zimmermann, K.; Haas, A.; Oxenius, A. Systemic Antibody Responses to Gut Microbes in Health and Disease. Gut Microbes 2012, 3, 42-47.

6. Abdulla, S.; Oberholzer, R.; Juma, O.; Kubhoja, S.; Machera, F.; Membi, C.; Omari, S.; Urassa, A.; Mshinda, H.; Jumanne, A. et al. Safety and Immunogenicity of RTS,S/AS02D Malaria Vaccine in Infants. N. Engl. J. Med. 2008, 359, 2533-2544.

7. Matsukura, N.; Onda, M.; Tokunaga, A.; Teramoto, T.; Fujita, I.; Okuda, T.; Yamashita, K. Detection of Serum IgG Antibody Against Helicobacter pylori from Childhood in a Japanese Population. J. Gastroenterol. 1994, 29, 403-405.

8. Coenen, D.; Verschueren, P.; Westhovens, R.; Bossuyt, X. Technical and Diagnostic Performance of 6 Assays for the Measurement of Citrullinated Protetn/Peptide Antibodies in the Diagnosis of Rheumatoid Arthritis. Clin. Chem. 2007, 53, 498-504.

9. Bizzaro, N.; Tonutti, E.; Tozzoli, R.; Villalta, D. Analytical and Diagnostic Characteristics of 11 2nd- and 3rd-generation Immunoenzymatic Methods for the Detection of Antibodies to Citrullinated Proteins. Clin. Chem. 2007, 53, 1527-1533.

10. Robinson, W. H.; DiGennaro, C.; Hueber, W.; Haab, B. B.; Kamachi, M.; Dean, E. J.; Fournel, S.; Fong, D.; Genovese, M. C.; de Vegvar, H. E. et al. Autoantigen Microarrays for Multiplex Characterization of Autoantibody Responses. Nat. Med. 2002, 8, 295-301.

11. Bakchoul, T.; Giptner, A.; Najaoui, A.; Bein, G.; Santoso, S.; Sachs, U. J. H. Prospective Evaluation of PF4/Heparin Immunoassays for the Diagnosis of Heparin-induced Thrombocytopenia. J. Thromb. Haemostasis 2009, 7, 1260-1265.

12. Jarius, S.; Probst, C.; Borowski, K.; Franciotta, D.; Wildemann, B.; Stoecker, W.; Wandinger, K. P. Standardized Method for the Detection of Antibodies to Aquaporin-4 
Based on a Highly Sensitive Immunofluorescence Assay Employing Recombinant Target Antigen. J. Neurol. Sci. 2010, 291, 52-56.

13. Waters, P.; Vincent, A. Detection of Anti-Aquaporin-4 Antibodies in Neuromyelitis Optica: Current Status of the Assays. Int. MS J. 2008, 15, 99-105.

14. Platt, G. W.; Damin, F.; Swann, M. J.; Metton, I.; Skorski, G.; Cretich, M.; Chiari, M. Allergen Immobilisation and Signal Amplification by Quantum Dots for Use in a Biosensor Assay of IgE in Serum. Biosens. Bioelectron. 2014, 52, 82-88.

15. Fitter, J.; Katranidis, A.; Rosenkranz, T.; Atta, D.; Schlesinger, R.; Büldt, G. Single Molecule Fluorescence Spectroscopy: A Tool for Protein Studies Approaching Cellular Environmental Conditions. Soft Matter 2011, 7, 1254-1259.

16. Nchimi-Nono, K.; Wegner, K. D.; Lindén, S.; Lecointre, A.; Ehret-Sabatier, L.; Shakir, S.; Hildebrandt, N.; Charbonnière, L. J. Activated Phosphonated Trifunctional Chelates for Highly Sensitive Lanthanide-based FRET Immunoassays Applied to Total Prostate Specific Antigen Detection. Org. Biomol. Chem. 2013, 11, 6493-6501.

17. Golynskiy, M. V.; Rurup, W. F.; Merkx, M. Antibody Detection by Using a FRET-Based Protein Conformational Switch. ChemBioChem 2010, 11, 2264-2267.

18. Liu, J.; Cheng, J.; Zhang, Y. Upconversion Nanoparticle Based LRET System for Sensitive Detection Of MRSA DNA Sequence. Biosens. Bioelectron. 2013, 43, 252-256.

19. Liu, Y.; Tu, D.; Zhu, H.; Chen, X. Lanthanide-Doped Luminescent Nanoprobes: Controlled Synthesis, Optical Spectroscopy, and Bioapplications. Chem. Soc. Rev. 2013, 42, 6924-6958.

20. Van Veggel, F. C. J. M.; Dong, C.; Johnson, N. J. J.; Pichaandi, J. Ln(3+)-Doped Nanoparticles for Upconversion and Magnetic Resonance Imaging: Some Critical Notes on Recent Progress and Some Aspects to be Considered. Nanoscale 2012, 4, 7309-7321. 
21. Casanova, D.; Giaume, D.; Gacoin, T.; Boilot, J. P.; Alexandrou, A. J. Single LanthanideDoped Oxide Nanoparticles as Donors in Fluorescence Resonance Energy Transfer Experiments. J. Phys. Chem. B 2006, 110, 19264-19270.

22. Dawson, J. F.; Holmes, C. F. B. Molecular Mechanisms Underlying Inhibition of Protein Phosphatases by Marine Toxins. Front. Biosci. 1999, 4, 646-658.

23. Jang, D. -J.; Guo, M.; Wang, D. J. Proteomic and Biochemical Studies of Calcium- and Phosphorylation-Dependent Calmodulin Complexes in Mammalian Cells. Proteome Res. 2007, 6, 3718-3728.

24. Valdiglesias, V.; Laffon, B.; Pásaro, E.; Méndez, J. Okadaic Acid Induces Morphological Changes, Apoptosis and Cell Cycle Alterations in Different Human Cell Types. J. Environ. Monit. 2011, 13, 1831-1840.

25. Diamente, P. R.; Burke, R. D.; van Veggel, F. C. J. M. Bioconjugation of Ln3+-doped LaF3 Nanoparticles to Avidin. Langmuir 2006, 22, 1782-1788.

26. Mattoussi, H.; Mauro, J. M.; Goldman, E. R.; Anderson, G. P.; Sundar, V. C.; Mikulec, F. V.; Bawendi, M. G. Self-Assembly of CdSe-ZnS Quantum Dot Bioconjugates Using an Engineered Recombinant Protein. J. Am. Chem. Soc. 2000, 122, 12142-12150.

27. Armstrong, J. K.; Wenby, R. B.; Meiselman, H. J.; Fisher, T. C. The Hydrodynamic Radii of Macromolecules and Their Effect on Red Blood Cell Aggregation. Biophys. J. 2004, 87, $4259-4270$.

28. Calzolai, L.; Franchini, F.; Gilliland, D.; Rossi, F. Protein-Nanoparticle Interaction: Identification of the Ubiquitin-Gold Nanoparticle Interaction Site. Nano Lett. 2010, 10, 3101-3105.

29. Menéndez, G. O.; Pichel, M. E.; Spaqnuolo, C. C.; Jares-Erijman, E. A. NIR Fluorescent Biotinylated Cyanine Dye: Optical Properties and Combination with Quantum Dots as a Potential Sensing Device. Photochem. Photobiol Sci. 2013, 12, 236-240. 
30. D’Hooge, F.; Elfeky, S. A.; Flower, S. E.; Pascu, S. I.; Jenkins, A. T. A.; van den Elsen, J. M. H.; James, T. D.; Fossey, J. S. Biotinylated Boronic Acid Fluorophore Conjugates: Quencher Elimination Strategy for Imaging and Saccharide Detection. RSC Adv. 2012, 2, 3274-3280.

Table of Contents Graphic

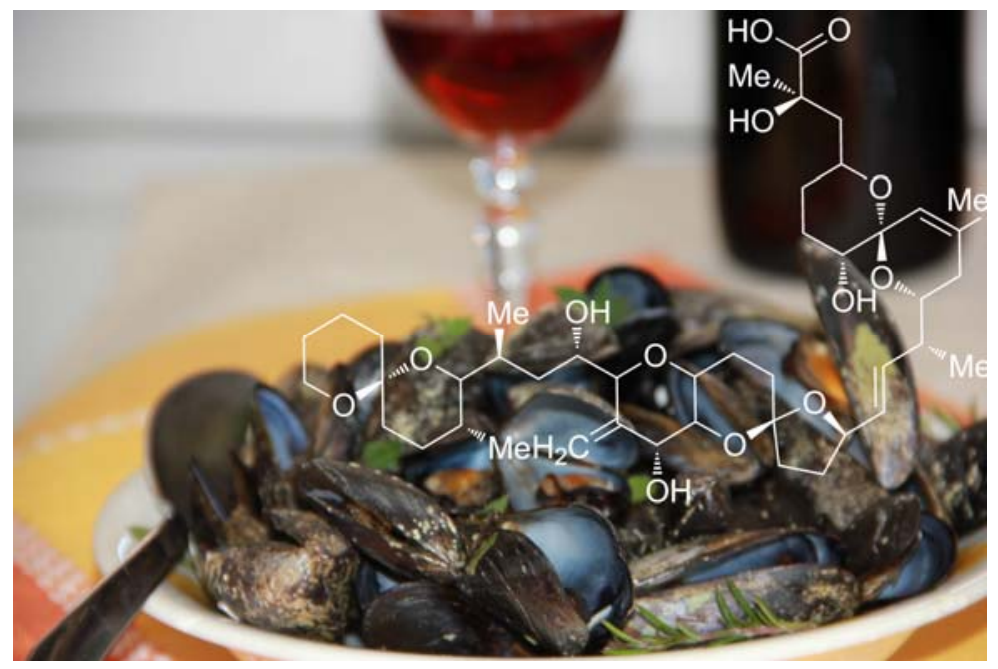

